Automation and Robotics in Construction $X$

G.H. Watson, R.L. Tucker and J.K. Walters (Editors)

(C) 1993 Elsevier Science Publishers B.V. All rights reserveci.

The Study on The Development of Building Construction Robots in Japan

Fumio Shintani and Masaki Yamamoto Senior Consultant, Technology Department, Integrated Research Division,
The Japan Research Institute, Limited

Kioi-cho Building, 3-12, Kioi-cho, Chiyoda-ku, Tokyo, 102, Japan

Abstract

At The Ninth ISARC held in Tokyo, we introduced thirteen construction robots for the building construction being developed by "Construction progress of the development of each robots and the possible program for the robots to be used at building construction sites.

1. ROBOTS UNDER DEVELOPMENT (Figure.1)

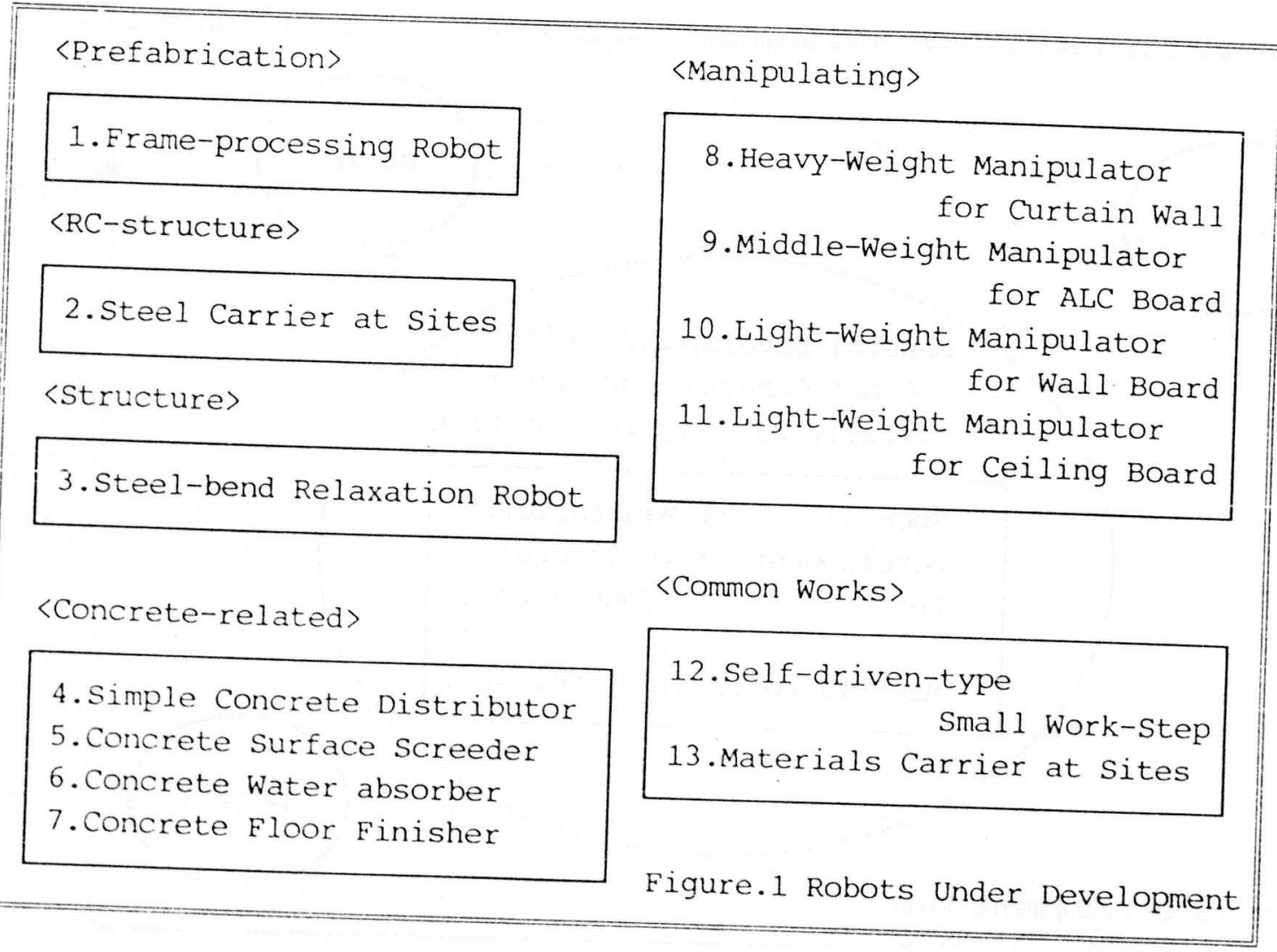




\section{THE WAY OF DEVELOPMENT}

We have taken the unique method for collaborative development. So far, development of construction robots has been conducted by each general contractors independently and this method resulted in the delay of development mainly because, in most cases, they could not create the market of construction robots for the robot manufacturers. This matter is very important because if there is no supplyer there is no possibility for construction robots to exist.

Thus, we organized ten groups for development of construction robots and each group consists of one manufacturer and twenty-nine general contractors. What is good with this method is that we can eliminate the market limitation caused by the independent development of one general contractor. We named group for development as a "Development Unit (DU)", and substantial development has been conducted by each Development Unit shown in Figure.2. below;

The members of each Development Unit agreed with the basic policy as

(1) Manufacturer is responsible for the development cost.

(2) General contractors are responsible for setting up the site for test.

(3) Intellectual property is basically owned by manufacturers.

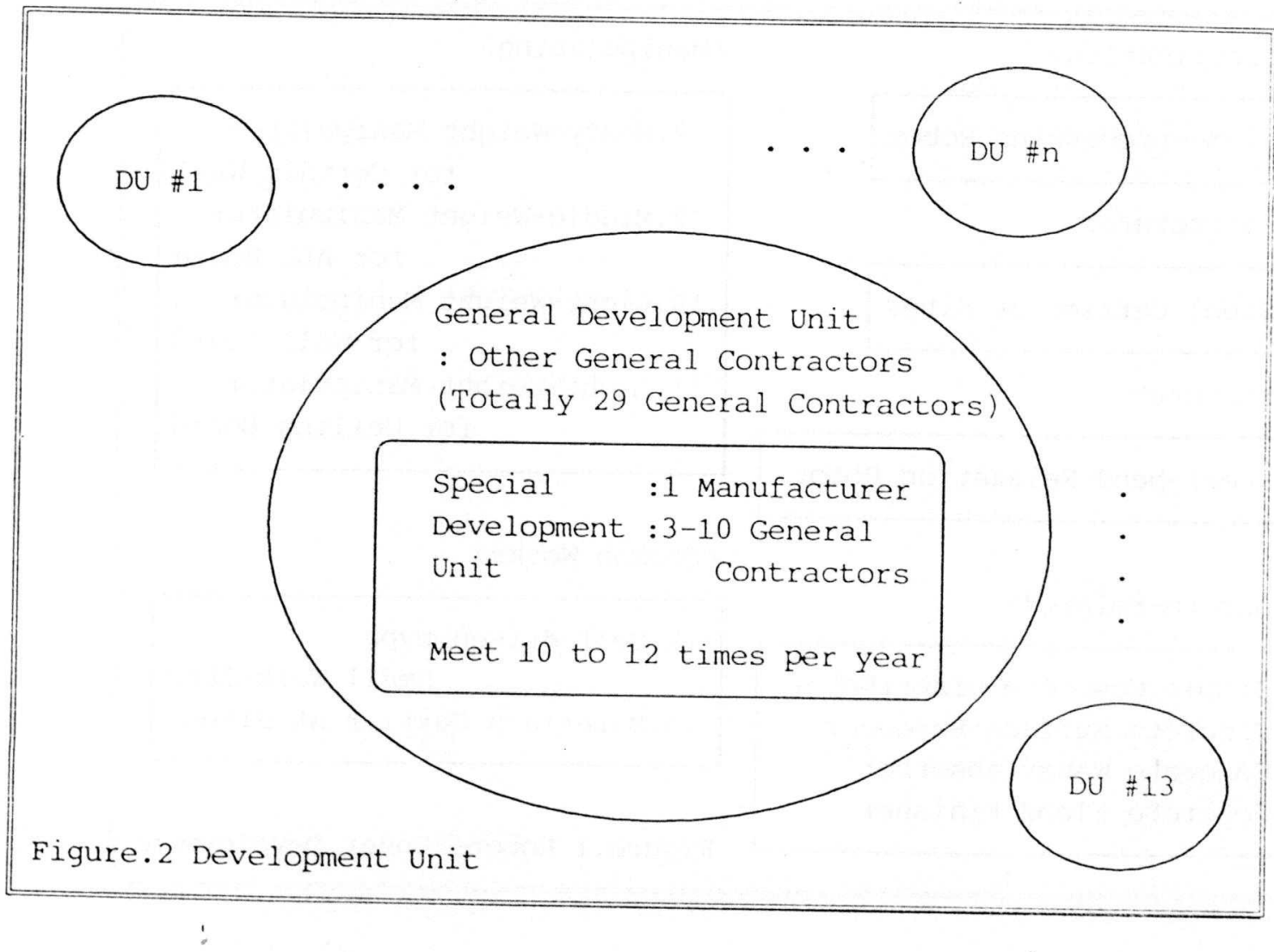




\section{PROGRESS OF THE DEVELOPMENT}

Consrtium project is now in third phase to introduce the proto-type robots to actual construction site. In first phase, we studied what kinds of robots are needed at construction sites and in second phase, we organized ten Development Units and started designing the specification of thirteen construction robots.

Because of the characteristics of each construction robots, progress of the development of each robots are in various stages as shown in Figure.3. However, most of robots are scheduled to be demonstrated by the end of this year because demonstration in front of users of construction robots is the one big target of Construction Robots Consortium.

One exception of the schedule mentioned above is "Frame-processing Robot". This is the prefabrication system which provides wood frames to mold concrete structure such as column and floor of (S)RC buildings instead of cutting wood plate at site. Actually this system is ready to be produced, however, it may cost two million dollars so that it is necessary to arrange the governmental support or simillar support to introduce this system into sub-contractors of carpenter work. Getting support is now under processing in the collaboration with governmental agency who is responsible for incubation of small companies.

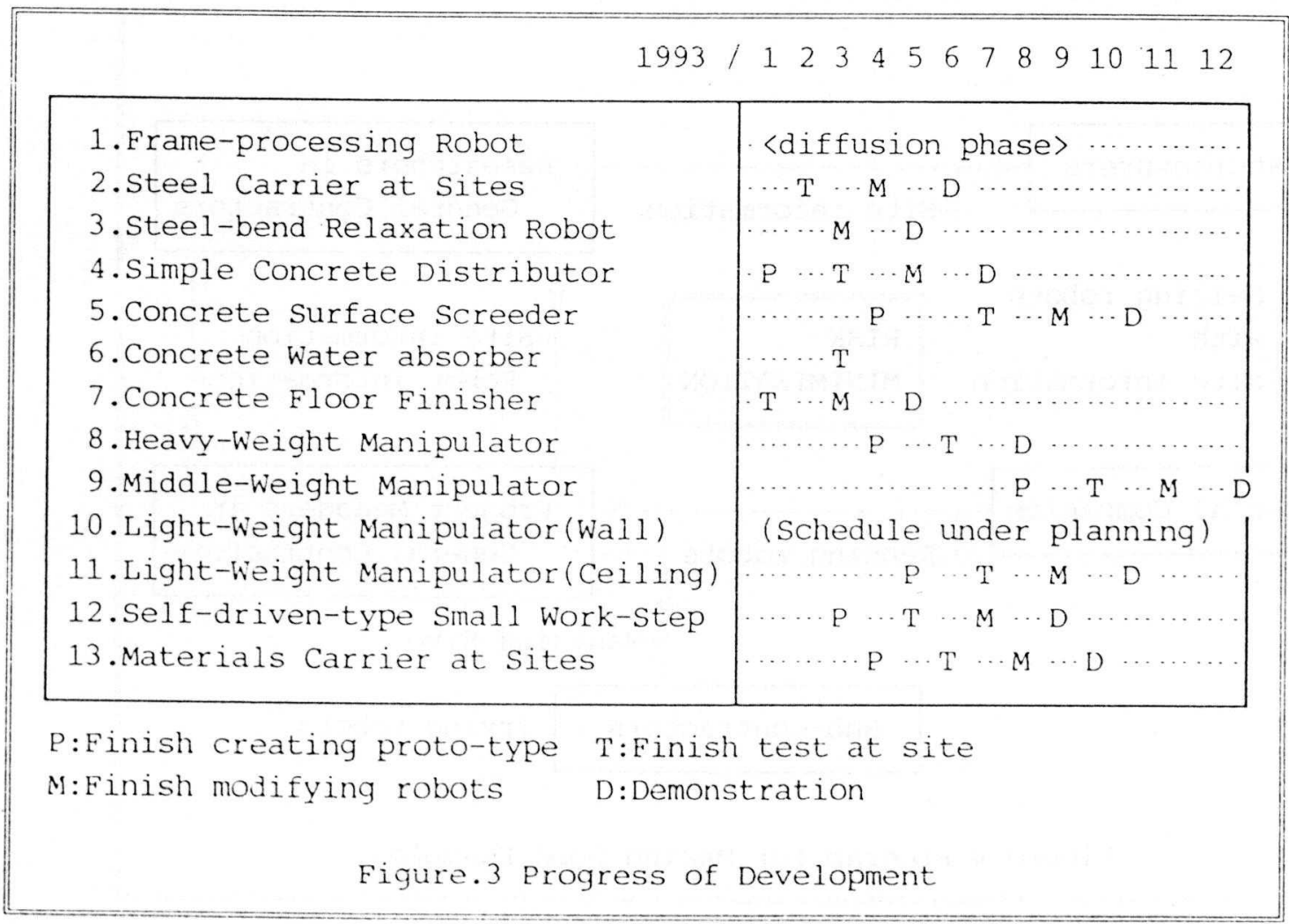




\section{PROGRAM FOR DIFFUSION OF CONSTRUCTION ROBOTS}

After the development it is important to introduce construction robots to the actual construction site. To do this, we have to solve the problems regarding three matters described below;

(1) Cost accumulation of building construction is based on the rate of human workers so that there is no measure for the work by robots.

(2) Most sub-contractors, who are the real users of construction robots, can not afford to buy construction robots because of financial reason.

(3) Recentiy, few general contractors own machines (includes construction robots) but use the lease/rental system when they need machines.

Thus we have two programs to introduce construction robots into actual building construction sites. First program is to make the standard of cost accumulation when using robots. We have started exploratory meeting with Ministry of Construction for possible system which enhance the automization. Second program is to make good examples of using construction robots at actual sites (Figure.4).

\section{CONCLUSION}

As I reported, development of constrcution robots by the consotium is now in final effort. Result will be reported next year.

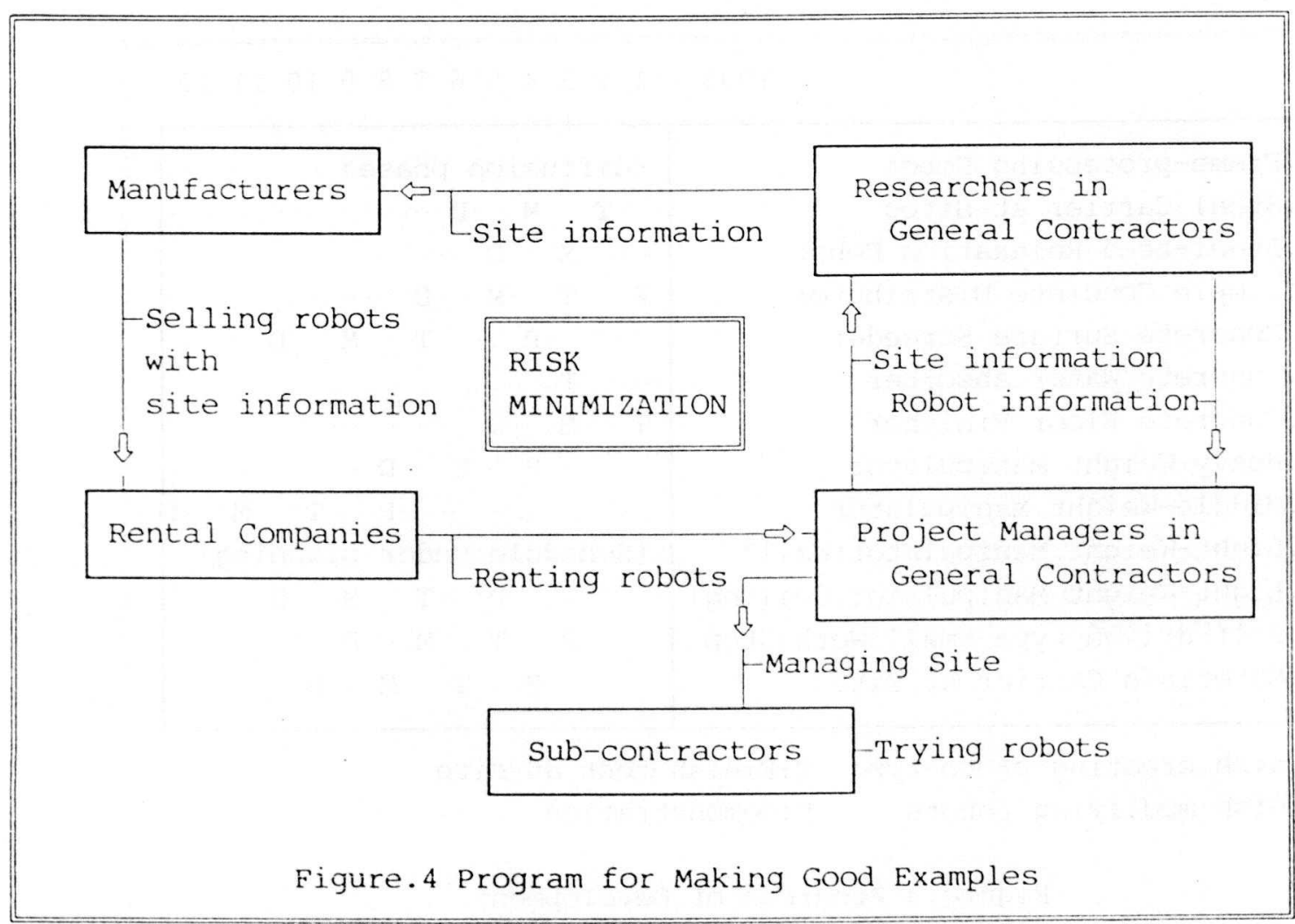

